\title{
Programa de intervención sustentado en el aprendizaje estratégico para orientar el proceso de composición escrita en estudiantes de educación superior
}

\section{Sustained intervention program in strategic learning to guide the written composition process in higher education students}

\author{
Carmen Alida Flores \\ carmenalidaf@yahoo.com
}

Universidad Pedagógica Experimental Libertador, Venezuela

\section{RESUMEN}

- Artículo recibido en septiembre 2018 - Arbitrado en octubre 2018 - Publicado en enero 2019

El desconocimiento metacognitivo que los estudiantes del nivel superior demuestran para componer un texto escrito constituyó el problema central en esta investigación. De allí que se haya propuesto un programa de intervención sustentado en el aprendizaje estratégico para orientar el proceso de composición escrita. La metodología estuvo basada en un diseño de investigación de campo de carácter descriptivo e interpretativo, apoyada, principalmente, en la metodología cualitativa y, complementada, con el enfoque cuantitativo de investigación. Se aplicó un cuestionario y una entrevista en profundidad a los sujetos de la investigación. Los resultados demostraron que los estudiantes abordados solo poseían habilidades lingüísticas de tipo léxico - semántico y sintáctico de su lengua, pero carecían de habilidades estratégicas que les impedían introducirse a niveles profundos de la escritura. Este hallazgo permitió demostrar la importancia de conocer las debilidades o carencias que poseen los estudiantes a fin de diseñar estrategias adaptadas a sus necesidades.

Palabras clave: Composición escrita; texto escrito; aprendizaje estratégico; programa de intervención; metacognición y Educación Superior

\begin{abstract}
The metacognitive ignorance that the students of the superior level demonstrate to compose a written text constituted the central problem in this investigation. Hence, an intervention program based on strategic learning has been proposed to guide the written composition process. The methodology was based on a descriptive and interpretive field research design, mainly supported by qualitative methodology and complemented by the quantitative research approach. A questionnaire and an indepth interview were applied to the research subjects. The results showed that the students approached only possessed linguistic-semantic and syntactic linguistic skills of their language, but lacked strategic skills that prevented them from entering deep levels of writing. This finding allowed demonstrating the importance of knowing the weaknesses or deficiencies that students have in order to design strategies adapted to their needs.
\end{abstract}

Keywords: Written composition; written texts; strategic learning; intervention program; metacognition and Higher Education 


\section{INTRODUCGIÓN}

Entre las actividades humanas, aprender es una de las más antiguas. Desde que nace, el hombre siente la necesidad de experimentar muchas cosas: satisfacer sus necesidades, caminar, hablar, llamar los objetos por su nombre, entre otras. Posteriormente aprende a leer y a escribir; a dominar operaciones matemáticas y reglas de ortografía. Sin embargo, una de las acciones que más se le dificulta es aprender a aprender.

Aprender a aprender implica la capacidad de reflexionar en la forma como se aprende y actuar en consecuencia autorregulando el propio proceso de aprendizaje mediante el uso de estrategias flexibles y apropiadas (Díaz Barriga y Hernández, 2002). Para estos autores, uno de los objetivos en educación debe ser el de enseñar a los alumnos a que se vuelvan aprendices independientes y autorreguladores, capaces de aprender a aprender. Los estudiantes que han aprendido a aprender controlan sus procesos de aprendizaje, se dan cuenta de lo que hacen, captan las exigencias de las tareas, responden consecuentemente, emplean estrategias de estudio pertinentes para cada situación, valoran los logros obtenidos y corrigen sus errores. Esto es lo que se denomina autonomía en el aprendizaje.

Monereo (2004) define el término autonomía como la probabilidad que tiene el estudiante de autorregular su propio proceso de estudio y aprendizaje en función de los objetivos que persigue $y$ de las condiciones del contexto que determinan la consecución de ese objetivo. Estas características para un aprendizaje autónomo se corresponden con las que se le atribuyen al aprendizaje estratégico.

Por su parte, Ríos (2004) define el aprendizaje estratégico como "un proceso potencialmente consciente $\mathrm{e}$ intencional mediante el cual el sujeto organiza y modifica sus planes de acción en función de logros de determinadas metas de aprendizaje.” (p. 12). Esta cita pone de manifiesto que el proceso educativo requiere que nadie piense por otro, ni vea por otro, ni hable por otro, ni actúe por otro. Por esta razón es tan importante aprender a aprender, aprender a educarse.

$\mathrm{Al}$ reflexionar a partir de los planteamientos de estos autores, se puede analizar que quizás esto es lo que no ha considerado la educación: respetar la autonomía de todo estudiante. La escuela siempre ha pretendido imponer cómo debe ser el proceso de aprendizaje, las condiciones del contexto y las estrategias que debe desarrollar el aprendiz. Es hora ya de que los educadores se den cuenta de que más que cumplir con unos contenidos programáticos, es más importante considerar que el estudiante debe tener 
un papel activo y protagónico dentro del aprendizaje, debido a que cada uno aporta una comprensión personal diferente de lo que se enseña; es él quien da sentido a los materiales que procesa; por tanto, en el aprendizaje se debe considerar los contenidos, los procesos, las estrategias, las técnicas y los estilos de aprendizaje.

Es necesario mencionar que en los últimos años la concepción en las investigaciones sobre la escritura ha sufrido cambios puesto que, a principios de los años setenta, en la mayoría de los trabajos sobre escritura el objeto de estudio era el producto ya acabado (el texto escrito final) y se centraban en aspectos de organización sintáctica $y$ léxica. A partir de los noventa, empieza el interés por la actividad cognitiva que ejecuta quien escribe y, en consecuencia, por el proceso que sigue al escribir.

Por lo antes planteado, en la presente investigación se abordaron estrategias de aprendizaje que contribuyeran con la formación de individuos capaces de producir adecuados textos académicos. Para lograr esto, se diseñó un programa de intervención sustentado en el aprendizaje estratégico que oriente el proceso de composición escrita en estudiantes de Educación Superior. Se propuso con este programa integrar la producción y el análisis de textos en la enseñanza de todas las áreas, porque leer $\mathrm{y}$ escribir forman parte del quehacer profesional y académico y son los medios ineludibles para aprender los contenidos conceptuales de cualquiera disciplina.

La escritura se conceptualiza a menudo como un producto físico y estático: un libro, un informe, una carta, etc. Sin embargo, la composición escrita es una acción que se desarrolla a través del tiempo y en la mente del autor donde ocurren los procesos cognitivos de construcción de significados (Cassany, 2001).

El arte de la palabra escrita está presente en los diferentes escenarios de todos los niveles educativos. Se considera que es una actividad imprescindible dentro del currículo, dada la necesidad de expresar adecuadamente las ideas por escrito y su relación con todas las áreas del saber.

Escribir de forma ajustada a las condiciones de una situación de comunicación supone ejecutar un conjunto de actividades cognitivas complejas que se llevan a cabo en función del contexto social y cultural donde se enmarca la producción textual. Así se considera que texto y contexto son inseparables en cualquier situación de escritura.

En los últimos años ha crecido el interés por integrar los procesos cognitivos con el contexto sociocultural donde se inscriben los textos, con el fin de enseñar 
a construir textos ajustados a unas determinadas situaciones de comunicación. El proceso cognitivo de cualquier escrito no puede ser considerado único, sino que su concreción depende de la interpretación que el escritor hace de las condiciones particulares de cada situación de comunicación (Castelló, 1999).

La capacidad de producir textos escritos supone el desarrollo de la competencia comunicativa, es decir, la posibilidad de generar textos correctos y aceptables. Se entiende por competencia comunicativa escrita a la capacidad de usar el lenguaje escrito para comunicar diferentes propósitos adecuadamente en las diversas situaciones sociales y culturales (Cassany, Luna y Sanz, 2000). Esta competencia incluye otras habilidades que intervienen en la elaboración de todo tipo de textos. Por un lado, la habilidad lingüística que alude al conocimiento del sistema de la lengua y el modo de utilizarlo; por el otro, la habilidad discursiva que refiere a la capacidad de seleccionar el discurso adecuado a la intención del escritor y a la situación comunicativa y textual, que permite organizar un texto de la manera más adecuada según el tipo de discurso seleccionado.

A la luz de los planteamientos anteriores, es deber de la escuela propiciar la formación de un sujeto que sea capaz de comunicarse de una manera adecuada tanto en forma oral como escrita. La complejidad del proceso de escritura obliga a fomentar el desarrollo de las capacidades del alumno a partir del manejo de diversos tipos de textos: narrativo, descriptivo, expositivo $\mathrm{y}$ argumentativo.

Sin embargo, uno de los problemas en materia de lengua materna, a los que se enfrenta la escuela es que los alumnos escriben como hablan. Más concretamente, cuando presentan un informe escrito, es frecuente que dejen de considerar aspectos esenciales o lo hacen de una manera incomprensible, incoherente $y$ con el vocabulario informal que emplean en la oralidad. El área teóricamente encargada de resolver esta situación, Lengua, se dedica a aprendizajes formales y memorísticos sobre la estructura de la lengua, con ejercicios de respuesta única, dictados, copias sin sentido y penalizaciones continuas. El docente presupone que la enseñanza de la escritura ya se realizó en los primeros grados de escolaridad y que en los niveles más avanzados centra su interés en aspectos más conceptuales y menos funcionales (Cassany, 2001).

Por otra parte, existen personas que se expresan oralmente en forma correcta; pero manifiestan un nivel precario de producción escrita. Esto, cuando se trata de estudiantes universitarios es aún más preocupante porque resulta inadmisible que en este nivel existan alumnos que 
presenten fallas de escritura y, lo que es más grave, que desconozcan la importancia de expresarse correctamente por escrito (Flores, 2001).

El alumnado escribe frecuentemente tanto en las áreas de las ciencias sociales: lengua, filosofía, sociología; como en las áreas de ciencias: matemática, física, química. Escriben textos académicos: apuntes, resúmenes, comentarios, informes. Sin embargo, las prácticas didácticas de enseñanza de la producción textual se circunscriben estrictamente al área de lengua materna. En fin, los estudiantes escriben muchos textos académicos, pero no se les orienta a cómo abordar el proceso de componer tales textos. Aunado a esto, la fealdad de los borradores está considerada como negativa. Los textos con tachaduras y correcciones sobre sí mismo son vergonzosos para quien los escribe y para quien los lee; la escuela enseña que los textos deben ser consignados de una manera impecable y así tendrá mayor ponderación. Esto fomenta la idea de que los textos publicados no tuvieron versiones intermedias y que sus autores los generaron en forma espontánea y sin esfuerzo.

Es posible que la causa de las dificultades que presentan estos alumnos para abordar la composición escrita esté en el poco énfasis en el desarrollo de estrategias de aprendizaje, en aprender a resolver problemas, aprender a pensar o aprender a aprender.

Díaz Barriga y Hernández (2002) explican que la mayoría de los estudiantes presentan dificultades para redactar un texto por cuanto: a) empiezan a escribir en forma casi inmediata, realizando muy pocas actividades relacionadas con la planificación; b) no saben estructurar una red jerárquica de metas y submetas en la planificación; c) aunque poseen un conocimiento de las características y elementos estructurales de algunos géneros como el narrativo, el expositivo y el argumentativo frente a tareas de composición, no son capaces de utilizarlos estratégicamente; d) sus producciones poseen poca riqueza e integración conceptual de las ideas y tienen un estilo segmentado, es decir, poco cohesionado; y e) sus escritos no están dirigidos a una determinada audiencia o lector, sino más bien a la subjetividad de su propio pensamiento.

En fin, los problemas en la redacción giran en torno a tres componentes esenciales: la planificación, la redacción propiamente dicha y la revisión.

Castelló (1999) señala que las dificultades de los adolescentes para producir textos coherentes y ajustados a diferentes situaciones comunicativas permiten suponer que muchas situaciones de enseñanza no favorecen el 
aprendizaje de un proceso reflexivo de composición. Por tanto, en la actualidad hay un problema sin resolver: explicar cómo se puede favorecer el paso de una escritura lineal, poco reflexiva, a un trabajo de composición más reflexivo, donde el escritor sea capaz de regular su producción en función de su interpretación y ajuste a las características comunicativas. Todo esto obedece a que quien escribe solo difunde versiones finales de sus textos, es decir, destruye el material intermedio de la composición: listas, esquemas, borradores, notas, apuntes, correcciones.

A los estudiantes del nivel superior se les dificulta redactar ideas, relacionar conceptos, parafrasear cualquier texto escrito, aplicar correctamente la ortografía, la morfología, la sintaxis y la puntuación; además de adecuar el escrito al contexto o audiencia destinada. $\mathrm{Su}$ competencia comunicativa ante la posibilidad de generar textos correctos y aceptables se muestra muy limitada, por cuanto si bien es cierto que estos estudiantes tienen dominio de la habilidad lingüística que alude al conocimiento del sistema de la lengua, también es cierto que muestran ausencia de la habilidad discursiva que refiere a la capacidad de seleccionar el discurso adecuado a la intención del escritor y a la situación comunicativa y textual, que permite organizar un texto de la manera más adecuada según el tipo de discurso seleccionado.
La situación anteriormente planteada, la mayoría de las veces, obstaculiza el rendimiento estudiantil porque si un alumno no se expresa correctamente por escrito, no puede responder de manera eficiente a las exigencias de cada asignatura, lo que redunda en un progreso lento en sus estudios y una permanencia excesiva en la carrera y, hasta podría pensarse, en un fracaso en ella.

Cuando se detalla lo que el alumno debe escribir: destinatario, extensión, registro, tema y estructura se convierte al aprendiz en un títere textualizador que ejecuta órdenes. El docente, por su parte, es a la vez víctima y responsable de ese conjunto de representaciones. Es víctima porque como miembros de la comunidad mantienen las mismas concepciones tradicionales en la enseñanza y en el aprendizaje de la lengua escrita y es responsable por su condición de transmisor y guía en la formación de sus alumnos.

Por tanto, en el proceso de composición escrita, el docente es quien decide qué es lo que se tiene que escribir, cómo y cuándo y la corrección es su responsabilidad, pues es él quien debe corregir todos los errores del texto a fin de que quede perfecto. Ante esta situación, el rol del alumno se limita a seguir las instrucciones del docente; no está capacitado para autocorregirse y es penalizado cuando comete errores. En 
fin, el alumno asume poca responsabilidad en el proceso global del trabajo.

Ante esta problemática y con el interés de aportar solución al problema planteado, la presente investigación se propuso dar respuestas a los siguientes objetivos:

1. Analizar las concepciones sobre el proceso de composición escrita que tienen los estudiantes de Educación Superior.

2. Diseñar un programa de intervención fundamentado en el aprendizaje estratégico para abordar el proceso de composición escrita en Educación Superior.

\section{MÉTODO}

Trabajar con la escritura implica abordar el problema desde varias dimensiones. Especialmente, si interesa la escritura desde una práctica social, requerirá de un enfoque descriptivo e interpretativo. La metodología seleccionada así lo plantea a continuación.

En este estudio se abordó la realidad de múltiples maneras. De allí que se desarrolló con una metodología que incluyera a los paradigmas cuantitativo y cualitativo; pues se postula la idea de que existen muchas alternativas metodológicas para conocer lo social, el fenómeno humano y los procesos que ocurren en la sociedad.

Hernández, Fernández y Baptista (2004) explican que para trabajar con la integración de los enfoques cualitativo y cuantitativo, se plantea el llamado modelo de enfoque dominante; en donde "el estudio se desarrolla bajo la perspectiva de alguno de los dos enfoques, el cual prevalece, y la investigación mantiene un componente del otro enfoque” (p. 20).

Esto se explica, por cuanto la investigación se enfocó cualitativamente con un componente cuantitativo: se utilizó una entrevista a estudiantes universitarios, sesiones de grupo con discusión abierta sobre las actividades, registros de notas y un cuestionario que fue administrado inicialmente a estos estudiantes para medir sus concepciones sobre el proceso de composición de un texto escrito.

Con respecto al diseño de investigación, este estudio constituyó una investigación de campo, porque la información fue recogida en la realidad donde se produjo; más concretamente, en los escenarios del Instituto Pedagógico de Miranda José Manuel Siso Martínez (IPMJMSM) correspondiente a la Universidad Pedagógica Experimental Libertador (UPEL); específicamente con estudiantes inscritos en un curso del área de Lengua y Literatura donde se 
producen textos académicos escritos; sin descontextualizar los datos aislándolos de su contexto natural. Esto permitió recoger la información de la manera más completa y fiel posible.

Por otra parte, este trabajo responde a las características de una investigación de campo, de carácter descriptivo e interpretativo, debido a que se registró la información ordenadamente del hecho investigado. Para Erickson (1999) este tipo de estudio "exige ser cuidadoso y reflexivo para describir los acontecimientos cotidianos en el escenario de trabajo y para identificar el significado de las acciones de esos acontecimientos desde los diversos puntos de vista de los actores" (p. 199). Por consiguiente, todos los datos recogidos en esta investigación fueron sometidos a un arduo y sistemático proceso descriptivo y de interpretación, los cuales aportaron información para el diseño posterior del programa de intervención en composición escrita.

Los participantes de la investigación fueron 18 estudiantes que cursan la carrera de Educación en el Instituto Pedagógico de Miranda José Manuel Siso Martínez (IPMJMSM); los mismos estuvieron inscritos en el curso Aprendiendo a Redactar correspondiente al área de Lengua y Literatura.

Aprendiendo a Redactar se constituye en una Actividad Electiva de Extensión, ubicada en el quinto período académico del Componente de Formación General del Plan de Estudios; lo que significa que en ella se inscriben estudiantes de las diferentes especialidades que ofrece el IPMJMSM.

Una vez seleccionado el diseño de la investigación y conformado el grupo de participantes, se procedió a aplicar las técnicas e instrumentos.

Para la obtención de los datos, se aplicó la técnica de la encuesta con un cuestionario. La misma se acompañó de una entrevista de tipo cognitivo donde la investigadora interrogaba a los sujetos sobre sus concepciones sobre los procesos que activan cuando escriben. Las entrevistas fueron grabadas en cintas magnéticas y posteriormente trascritas para su análisis.

Se empleó, en algunos momentos, la técnica de los protocolos orales, es una técnica utilizada en las investigaciones sobre procesos cognitivos, y consiste en "hacer pensar en voz alta" a los sujetos mientras escriben o después de escribir inmediatamente. Los protocolos constituyen la descripción verbal que hacen los sujetos de sus procesos mentales (Arroyo y Mata, 2003).

Para el protocolo, se utilizó un cuestionario que ayudara a reflexionar a los estudiantes, pues se les dificultaba verbalizar sus pensamientos. 
Entre las técnicas empleadas en la aplicación del estudio están: la entrevista en profundidad y la observación participante. Los instrumentos empleados para recabar información fueron: un cuestionario, preguntas generadoras a manera de guión de entrevista, un grabador, notas de campo $\mathrm{y}$ autoinformes.

En la entrevista, se utilizaron preguntas generadoras redactadas por la investigadora previamente, para asegurar que los aspectos claves de la composición escrita fuesen explorados.

A través de la observación participante, se apreció la actividad verbal y no verbal de los participantes en situaciones de escritura en parejas o en pequeños grupos que, al exigir la toma de decisiones conjunta, favoreció la explicación del proceso seguido.

El cuestionario fue otro de los instrumentos empleados, así como las notas de campo y los autoinformes. Este último es la información que proporciona un sujeto sobre sí mismo o sobre distintos aspectos de su funcionamiento. Consistía, algunas veces, en breves escrituras; otras veces, protocolos de pensamiento en voz alta obtenidos simultánea e inmediatamente después de realizar una composición escrita. Los protocolos constituyen la descripción verbal que hacían los sujetos de sus procesos mentales.
El procedimiento se estructuró en etapas y sus correspondientes fases que permitieran recopilar toda la información necesaria para llevar a cabo los objetivos previamente planteados. La investigación se estructuró así en dos etapas.

\section{Etapa I. Estudio de la realidad}

En este lapso se realizaron diversas actividades que permitieron dar inicio a la investigación.

Fase 1: revisión exhaustiva de la teoría existente sobre el proceso de composición escrita a fin de determinar las variables que se pretendían medir a través de un instrumento que se diseñaría para su posterior aplicación.

Fase 2: iniciado el período académico 2017 - II (octubre de 2017), se aplicó el instrumento diseñado y validado previamente para la realización de la diagnosis que serviría de base para determinar las concepciones que tenían estos estudiantes sobre el proceso de composición escrita. Posteriormente, se les convocó a la semana siguiente, en el mismo horario, para una entrevista en profundidad sobre el mismo tópico. Las entrevistas fueron realizadas a cada uno de los sujetos participantes, por la autora de esta investigación con ayuda de dos profesoras especialistas en el área Lengua y Literatura. 
Fase 3: Destinado al ordenamiento de los datos. En esta fase, se contó con un gran volumen respuestas reflejadas en el cuestionario, cintas de audio y trascripciones de entrevistas. Todo esto se clasificó por orden de recolección y por las categorías de análisis que emergieron en esta investigación.

Fase 4: Se realizaron triangulaciones entre el cuestionario, la entrevista y la investigadora.

\section{Etapa II. Diseño del Programa de Intervención para el proceso de composición escrita (PROPCE)}

Fase 1: selección de los referentes teóricos que sustentaron el PROPCE, en función a las debilidades detectadas durante el desarrollo del diagnóstico y en relación con el proceso que se debe seguir para componer un texto escrito.

Fase 2: Proceso de planificación del programa. Se seleccionó el título, se tomaron decisiones en relación con el propósito, los objetivos, contenidos, duración y procedimientos. Se seleccionaron los recursos didácticos $\mathrm{y}$ las técnicas de recolección de información (evaluación de la efectividad del programa). Las sesiones fueron denominadas "Secuencias Didácticas” ,las cuales incluían estrategias instruccionales y estrategias de aprendizaje bajo el enfoque del aprendizaje estratégico.

El diseño de cada una de las secuencias didácticas estuvo fundamentado en la propuesta de Monereo (2002) y su enfoque del aprendizaje estratégico.

\section{RESULTADOS}

Se organizaron en las dos etapas desarrolladas en el procedimiento; estas son: Etapa I: Estudio de la realidad y etapa II: Diseño del programa de intervención para el proceso de composición escrita.

\section{Etapa I. Estudio de la realidad}

En esta etapa, se procedió al análisis de cada ítem del cuestionario los cuales fueron expuestos a un proceso de análisis estadístico, a través del programa computacional o paquete estadístico SPSS. Versión 12.0 de Microsoft.

Los resultados anteriores demuestran, después de un minucioso análisis y para dar respuestas al primer objetivo de esta investigación, que se pudo determinar cuáles son las concepciones que tienen los alumnos universitarios sobre el proceso de composición escrita, las cuales se detallan en la Figura 1: 
Escribir se reduce a registrar, copiar, almacenar información

Su única finalidad al escribir es por obligación, para cumplir los deseos del profesor

Los textos se construyen con muchas ideas

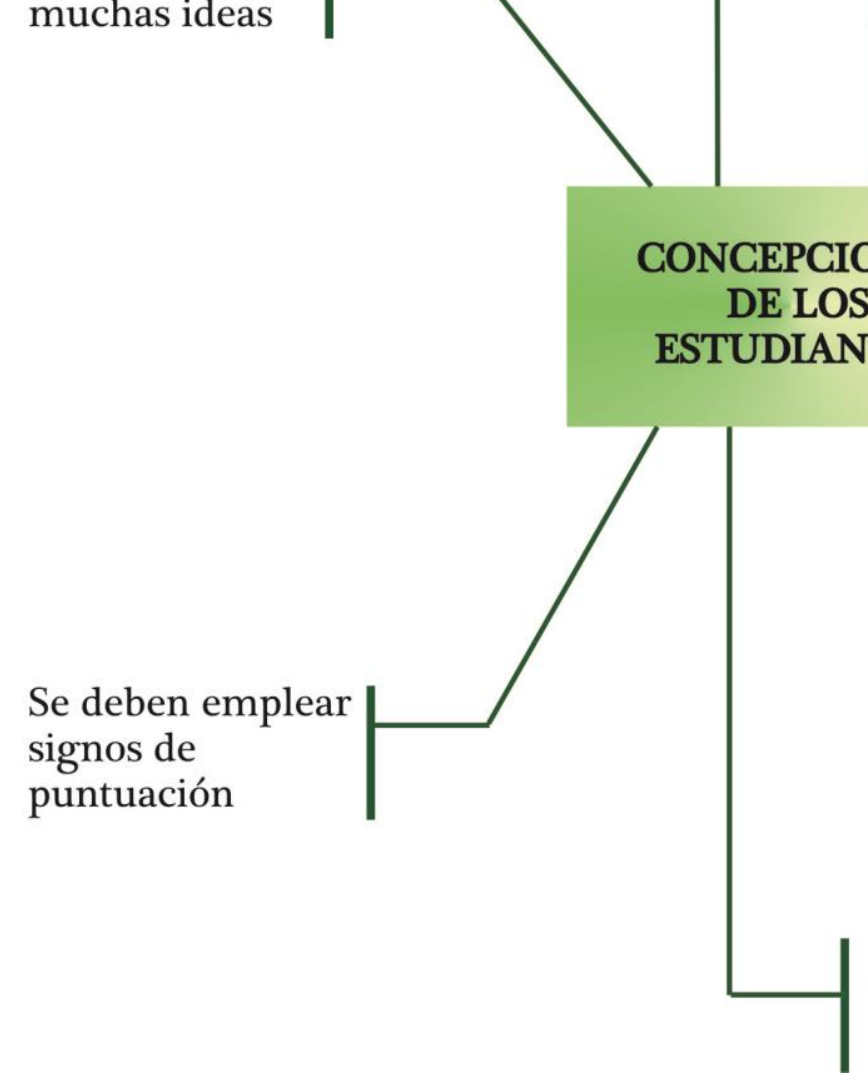

Se deben consultar

diversas fuentes

Figura 1. Concepciones de los estudiantes

Lo anterior muestra lo que los alumnos saben, mas no significa que esto es lo que hacen.

En la Figura 2, se presentan las concepciones que deben aprender los estudiantes de Educación Superior. 


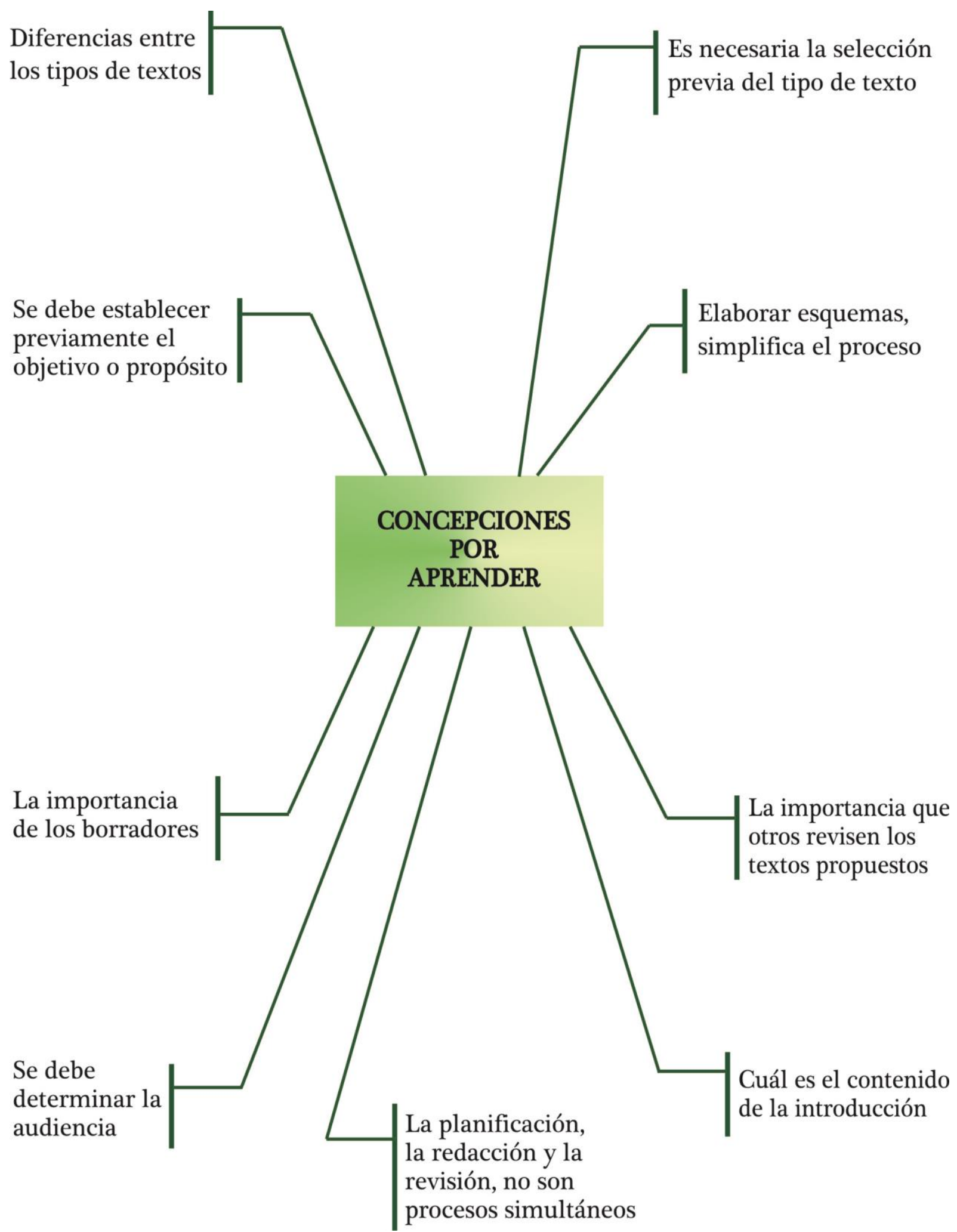

Figura 2. Concepciones por aprender 
A partir de este análisis es posible trazar una aproximación a las concepciones de los estudiantes sobre la escritura en el contexto académico. En primer lugar, en sus respuestas y su actuación se desprende una clara diferenciación entre su "saber" y su "hacer"; por ejemplo, la mayoría de los estudiantes universitarios poseen algunos conocimientos como la organización en introducción, desarrollo y conclusión. Sin embargo, rara vez incluyen los tres aspectos en sus textos escritos.

Para Castelló (1999), se hace necesario las relaciones e interacciones entre los diferentes conocimientos sobre el proceso (actividad cognitiva), sobre los objetivos que guían las tareas (finalidad discursiva), sobre diferentes situaciones comunicativas y características o propiedades lingüísticas (gramática, recursos estilísticos, géneros textuales, destinatarios) y el conocimiento específico que el escritor posee sobre el tema sobre el cual va a escribir. En fin, los estudiantes saben sobre escritura, pero no ejecutan el proceso de composición escrita.

\section{Etapa II. Diseño del Programa de Intervención para el proceso de composición escrita (PROPCE)}

En esta etapa de la investigación, se diseñó el Programa de Intervención para el Proceso de Composición Escrita (PROPCE). Para ello se seleccionaron los referentes teóricos que sustentarían este programa. Tales referentes giran en torno a la enseñanza estratégica.

\section{Programa de Intervención para el Proceso de Composición Escrita (PROPCE)}

Para escribir en texto hace falta, según Castelló (1999), cuatro tipos de conocimientos previos: a) conocimiento sobre el código; b) cantidad y tipo de conocimiento lingüístico -gramaticales: concordancia, sintaxis, conectores, géneros, etc.; c) conocimiento acerca del tema sobre el que se va a escribir; y d) conocimiento sobre el proceso de composición de la escritura.

La escuela ha hecho énfasis en los tres primeros conocimientos planteados por Castelló (1999); de allí que el presente programa de intervención enfatice en el cuarto y último conocimiento. Se considerarán, por tanto, una intensa actividad de reflexión sobre el proceso que se va a seguir y la relectura del texto producido.

Por otro lado, a través del PROPCE se propicia en los estudiantes el desarrollo de estrategias, sin limitarse a la memorización, sino avanzar a procesos cognitivos de alto nivel como la resolución de problemas y la toma de decisiones; es decir, se hará énfasis en el aprender a aprender o el aprendizaje 
estratégico como forma de seguir aprendiendo a lo largo de toda la vida.

Se entiende al aprendizaje estratégico como el proceso potencialmente consciente e intencional mediante el cual el sujeto organiza y modifica sus planes de acción en función de logros de determinadas metas de aprendizaje (Ríos, 2004).

Enseñar estrategias de aprendizaje implica, pues enseñar a reflexionar sobre las condiciones cambiantes de cada escenario y ajustar la propia actuación a estas condiciones. De allí que en una enseñanza estratégica de la composición escrita lo más importante serán los borradores de lo que produzca quien escribe.

\section{Propósito}

El propósito de este programa consiste en orientar el proceso de composición escrita para aprender a construir textos académicos desde una perspectiva retórica, discursiva y funcional dentro de un contexto comunicativo. El presente programa adquiere importancia por cuanto es necesario garantizar la enseñanza del proceso de composición escrita en el área de lengua y su transferencia al resto de las áreas curriculares donde la escritura es un instrumento mediatizador del conocimiento. Se pretende con este programa desarrollar un trabajo de composición más reflexivo, donde el escritor sea capaz de regular su producción en función de su interpretación y ajuste a las características comunicativas.

\section{Participantes}

El programa está dirigido a estudiantes de Educación Superior en las diferentes especialidades y carreras.

\section{Objetivo general}

Aprender el proceso de composición escrita de textos académicos desde una perspectiva retórica, discursiva $\mathrm{y}$ funcional ajustados a una determinada situación de comunicación.

\section{Objetivos específicos}

1. Los alumnos explicarán la estructura y características de los diferentes órdenes del discurso.

2. Analizar los conocimientos que debe manejar el escritor sobre el tema a escribir.

3. Establecer las diferencias entre un escritor experto y un escritor novato.

4. Aumentar la conciencia del alumno sobre las operaciones y decisiones mentales que se deben seguir para componer un texto escrito y valorar sus propias competencias $y$ debilidades.

5. Abordar la composición escrita de textos académicos desde un enfoque 
integrador sociocognitivo el cual explica cómo se puede favorecer el paso de una escritura lineal, poco reflexiva, a un trabajo de composición más reflexivo, donde el escritor sea capaz de regular su producción en función de contextos comunicativos específicos.

6. Los estudiantes producirán un texto escrito siguiendo el modelo de la enseñanza estratégica.

\section{Bases teóricas que sustentan el modelo}

La psicología cognitiva con Ausubel (1976), las concepciones de Vygostky (1977), la lingüística textual de van Dijk (1980), los órdenes discursivos con Sánchez (1993) y el aprendizaje estratégico con Monereo (2004).

\section{Metodología}

La metodología a seguir se sustentó en la que corresponde a un taller; por tanto, es totalmente participativa. Se esperaba que a través de la reflexión y del análisis de los trabajos realizados, los estudiantes adquirieran los contenidos. Se desarrolla cada taller a través de la aplicación de estrategias instruccionales y el desarrollo de estrategias de aprendizaje.

La asistencia, la participación, los ejercicios propuestos y la elaboración de los trabajos fueron indispensables para garantizar la consecución de los objetivos.

El trabajo individual y no presencial consistió en la realización de una carpeta contentiva con las evidencias de logro de los objetivos propuestos $\mathrm{y}$ en la elaboración de un diario personal (autoinforme) de cada sesión que permita la reflexión.

La evaluación tuvo en cuenta tanto el proceso como el producto final realizado. Por ello, se recopilaron en una carpeta (o portafolios) todo tipo de evidencias del progreso obtenido; en ella se reflejaban la evolución y el aprovechamiento del taller por parte de cada estudiante.

\section{Tiempo estimado}

El programa se diseñó para trabajar en ocho sesiones presenciales de dos horas cada una, para un total de 16 horas y algunas horas de asesorías individuales.

\section{Procedimientos para enseñar los contenidos}

Se desarrolló el programa siguiendo las fases de la enseñanza estratégica propuestas por Monereo (2004): a) fase 1. Presentación de la estrategia; b) fase 2. Práctica guiada de la estrategia; y c) fase 3. Práctica autónoma de la estrategia. 
A nivel metodológico, el estudio consiste en analizar la actividad verbal y no verbal de los participantes en situaciones de escritura en parejas o en pequeños grupos que, al exigir la toma de decisiones conjunta, favorece la explicitación del proceso de composición escrita que se va a seguir. Esto pone de manifiesto el tipo de conocimiento que utilizan los alumnos.

El docente ofrecerá las ayudas necesarias para guiar el proceso. Estas ayudas permiten: activar procesos cognitivos (planificar, revisar las decisiones tomadas) y ser retiradas las ayudas a medida que los estudiantes las interioricen y sean capaces de gestionar de manera autónoma su propio proceso de composición.

Se debe también trabajar el conocimiento sobre el propio proceso de composición: Analizar aquello que los estudiantes sean capaces de explicar sobre su propio proceso de composición escrita; es decir, que ellos mismos expliquen el proceso seguido para producir un texto. Se les formularán interrogantes como: ¿Cómo has hecho para escribir este texto?, ¿cuáles son las tareas de escrituras que actualmente realizas en el contexto académico?, ¿por qué las realizas?, ¿cuál es desde tu punto de vista el objetivo de estas tareas?, ¿qué haces para resolverlas?, ¿qué estrategia pones en marcha? Todo este procedimiento estará regido por la autorregulación que debe desarrollar los estudiantes.

\section{Secuencias didácticas}

Las secuencias didácticas explicitadas a continuación serán presentadas agrupando los talleres por bloques de contenidos: La primera secuencia didáctica incluye los talleres 1 y 2 ; la segunda secuencia didáctica solo presenta el 3 taller y la tercera secuencia didáctica incluye los talleres $4,5,6,7$ y 8 .

\section{Primera secuencia didáctica (talleres 1 y}

2). Contenido: estructura de los órdenes del discurso (narrativo, descriptivo, expositivo y argumentativo.

Se inició este enfoque con el análisis de las concepciones sobre escritura que tienen los alumnos y del proceso de composición. Se entregó un material mimeografiado el cual incluyó las características de los órdenes del discurso. Antes de leer el material, se explicó los tipos de conocimientos previos que se deben activar en el proceso de escritura: a) conocimiento sobre el código de la lengua; b) conocimiento lingüístico (gramatical: concordancia, sintaxis, conectores, tipos de textos, etc.); c) conocimiento acerca del tema sobre el que se va a escribir; y d) conocimiento sobre el proceso de composición escrita. En esta secuencia didáctica, se enfatizó en el conocimiento lingüístico específicamente en los 
órdenes del discurso. Además se trabajó la estructura de la introducción, desarrollo y conclusión.

\section{Estrategias de aprendizaje}

Aplicar la estrategia de elaboración, la cual según Elosúa y García (1993) supone integrar y relacionar información con los conocimientos previos pertinentes. En esta primera secuencia, no se desarrolló aún ningún procedimiento de autorregulación. Los estudiantes, a través de la síntesis como estrategia basada en procesos cognitivos, conformaron el tema sobre los órdenes del discurso en función de los elementos que lo integran y para la elaboración de conclusiones. Ejecutaron también ejercicios prácticos.

\section{Segunda secuencia didáctica (taller 3).}

Contenido: qué sabe el escritor sobre el tema. Solicitar a los alumnos la selección de una noticia de prensa diaria. Durante tres días, cada estudiante deberá adquirir la prensa diaria y recortar la noticia relacionada con el tópico previamente seleccionado. En clase, cada alumno mencionará la noticia que seleccionó y entre todos seleccionarán una de ellas. Se asignó la redacción de un texto escrito sobre el tema previamente seleccionado. Posteriormente, el docente invitó a los alumnos a conversar sobre el tema seleccionado. Después de debatido ampliamente el tema, nuevamente se les solicitó que escribieran un segundo texto relacionado con ese tema.

\section{Estrategias de aprendizaje}

En clase, cada uno de los alumnos compuso un texto cuyo tema fue seleccionado entre todos los artículos periodísticos traídos. Después de la discusión, nuevamente cada estudiante debió redactar el resumen del tema tratado en la discusión. La finalidad de esta actividad fue que los alumnos compararan la primera redacción con los pocos conocimientos que manejaban y la redacción cuando conocía el tema.

\section{Tercera secuencia didáctica (talleres 4,} 5, 6, 7 y 8). Contenido: diferencias entre expertos y novatos. La composición escrita como proceso. Etapas o momentos de la composición escrita: Planificación, redacción y revisión. Procesos cognitivos básicos en la composición de un texto expositivo: análisis, síntesis, comparación y la solución de problemas.

Se trabajó el conocimiento sobre el propio proceso de composición: analizando aquello que los estudiantes son capaces de explicar sobre su propio proceso de composición escrita; es decir, que ellos mismos expliquen el proceso seguido para componer un texto escrito. 
La aplicación o puesta en escena se dividió en dos grandes fases: (a) La composición dirigida: se incluyen aquí actividades donde el facilitador condujo el proceso de aprendizaje, con el fin de que se cumplan los pasos para asegurar con éxito la composición escrita.

La composición espontánea; se planifican actividades para que el alumno trabaje independientemente, el docente interviene cuando el alumno solicite ayuda. Esta puesta en escena se realizó en base a las fases de la enseñanza estratégica propuestas por Monereo (2004):

Fase I. Presentación de la estrategia: consistió en explicar el conjunto de decisiones que guiarían el proceso de aprendizaje de la composición escrita. Se trabajó la entrevista a un experto y el modelado.

Se hizo entrega de un material mimeografiado el cual explica paso a paso el proceso de composición escrita. En base a este contenido, se asignó la realización de una entrevista a un experto y una entrevista a un novato (se consideró experto a un docente del instituto, destacado como autor de artículos en revistas especializadas y se consideró novato a un estudiante compañero de la universidad).
Se discutieron en clase, los resultados de las entrevistas efectuadas y se establecieron conclusiones en base a diferencias entre expertos y novatos.

El modelado consistió en que la facilitadora del curso actuó como modelo construyendo un texto escrito en el pizarrón, explicando y justificando punto por punto todo lo que hacía $y$ pensaba mientras componía el texto.

Fase II. Acá se siguió la práctica guiada donde los alumnos practicaron la estrategia introducida en la etapa anterior y relacionada con el proceso de composición escrita. Se trabajó con los métodos de aprendizaje cooperativo y la interacción cara a cara. En el primero, se establecieron las diferencias que demuestran los alumnos en cuanto a conocimientos y habilidades; en el segundo, se favorecieron las relaciones interpersonales de ayuda, asistencia, soporte y refuerzo entre ellos. Estas actividades estuvieron reforzadas por la guía para la planificación, redacción y corrección de textos académicos.

Fase III. Práctica autónoma de la estrategia. En la última fase de la secuencia didáctica, los alumnos redactaron textos escritos estableciendo análisis, síntesis, comparaciones entre textos producidos antes y después del taller. 


\section{Estrategias de aprendizaje}

Diseñar un guión de entrevista a la luz de la guía "Proceso de composición escrita”.

Entre las estrategias cognitivas desarrolladas por los estudiantes en esta secuencia didáctica están: análisis e indagación de informaciones provenientes de la entrevista.

Redactaron textos escritos ajustados a la práctica guiada y posteriormente a la práctica autónoma.

\section{Evaluación}

El estudio consiste en analizar la actividad verbal y no verbal de los participantes en situaciones de escritura en parejas o en pequeños grupos que, al exigir la toma de decisiones conjunta, favorece la explicitación del proceso de composición escrita que se va a seguir. Esto pone de manifiesto el tipo de conocimiento que utilizan los alumnos: a) intensa actividad de reflexión sobre el proceso que se va a seguir; y b) la relectura del texto producido.

La evaluación presentada fue por carpetas o portafolios, donde se le otorgó mayor importancia a los borradores y a los autoinformes.

\section{CONCLUSIONES}

Las concepciones que manejaban los sujetos de este estudio, sobre el proceso de composición escrita resultaban insuficientes para abordar este proceso; más concretamente, solo poseían habilidades lingüísticas de tipo léxico semántico y sintáctico de su lengua, pero carecían de habilidades estratégicas que les impedían introducirse a niveles profundos de la escritura. Demostraron que hay una distancia entre lo que "saben" y lo que "saben hacer". En este sentido, esta investigación demostró que saben: formular un objetivo, qué es una audiencia, generar ideas, elaborar esquemas, redactar, que todo texto debe ser previamente corregido, entre otros; sin embargo, en la práctica demostraron que no ejecutan lo que saben para planificar, redactar y corregir un texto escrito. Los alumnos escriben de una forma automática, sin mucha reflexión ni elaboración.

El programa de intervención diseñado se ajustó a las debilidades de los estudiantes (fallaban en el empleo de estrategias), pues se logró nivelar sus conocimientos previos con el dominio de las habilidades estratégicas necesarias para abordar el proceso de composición escrita. A fin de lograr estas destrezas, se establecieron relaciones entre los conocimientos 
previos que poseían los alumnos y la información nueva presentada. Esto los ayudó a aprender a aprender, es decir aprendieron a construir conocimientos nuevos a partir de su relación con conocimientos ya existentes en su estructura cognoscitiva. Aprender a aprender equivale a ser capaz de realizar aprendizajes significativos por uno mismo en una amplia gama de situaciones y circunstancias.

En fin, los resultados obtenidos, permitieron demostrar la importancia de conocer las debilidades o carencias que poseen los estudiantes a fin de diseñar estrategias adaptadas a sus necesidades. A efectos de esta investigación, las carencias de los alumnos estuvieron en el dominio de estrategias cognitivas y metacognitivas para abordar la composición de textos académicos. En tal sentido, los resultados fueron altamente positivos por cuanto lograron alcanzar la composición de los textos académicos, orientados por el aprendizaje estratégico.

\section{REFERENCIAS}

Arroyo G., R. y Mata, F. S. (2003). El proceso de planificación en la composición escrita de alumnos de educación primaria. En Revista de Educación de la Facultad de Ciencias de la Educación Universitaria de Granada. 336 (2005), pp. 353 - 376

Ausubel, D. (1976). Psicología Educativa. México: Trillas
Cassany, D. (2001). Glosas didácticas. Barcelona: Universitat Pompeu Fabra

Cassany, D. Luna, M. y Sanz, G. (2000). Enseñar Lengua. Barcelona: Graó

Castelló B., M. (1999). El conocimiento que tienen los alumnos sobre la escritura. En J. I. Pozo y C. Monereo (Coord.). El Aprendizaje Estratégico. Madrid: Santillana Aula XXI

Díaz-Barriga, F. y Hernández, G. (2002). Estrategias docentes para un aprendizaje significativo. Una interpretación constructivista. $2^{\mathrm{a}}$ edición. México. Mc Graw - Hill Interamericana

Elosúa, M. R. y García, E. (1993). Estrategias para enseñar y aprender a pensar. Madrid: IEPS-Narcea

Erickson, F. (1999). Métodos Cualitativos de Investigación sobre la enseñanza. En La Investigación de la Enseñanza II. (Comp. Wittrock). Barcelona: Paidós

Flores, C. A. (2001). Estrategias instruccionales para la comprensión de lectura en estudiantes de Educación Integral en el Instituto Pedagógico de Miranda José Manuel Siso Martínez (trabajo de maestría). Universidad Pedagógica Experimental Libertador, Instituto Pedagógico de Miranda José Manuel Siso Martínez, Venezuela

Hernández S., R., Fernández C., C. y Baptista L., P. (2004). Metodología de la Investigación. (3ra. ed.). México: McGraw-Hill Interamericana

Monereo, C. (2002). Estrategias de Aprendizaje. Madrid: Visor

Monereo, C. (2004). La Enseñanza Estratégica: Enseñar para la autonomía. Revista Cándidus. 3 (27), $3-10$ 
Ríos C., P. (2004). Esbozo del enfoque estratégico del aprendizaje. Revista Cándidus. 3 (27), 11 - 15

Sánchez de R., I. (1993). Coherencia y órdenes discursivos. En Revista Letras. 50. CILLAB. IPC. UPEL

Van Dijk, T. (1980). Estructura y funciones del discurso. México: Siglo XXI
Vygostky, L. S. (1977). Pensamiento y Lenguaje. Teoría del Desarrollo Cultural de las Funciones Psíquicas. Buenos Aires: La Pléyade 